\title{
Arrhythmogenic Mechanism of the Enhanced Late Sodium Current in Human Ventricular Myocytes - a Simulation Study
}

\author{
Wei Wang ${ }^{1,2,3}$, Shanzhuo Zhang ${ }^{4}$, Yong Xu ${ }^{1}$, Kuanquan Wang ${ }^{4}$, Henggui Zhang ${ }^{2,3,4,5}$ \\ ${ }^{1}$ Harbin Institute of Technology, Shenzhen, China \\ ${ }^{2}$ Space Institute of Southern China, Shenzhen, China \\ ${ }^{3}$ Peng Cheng, Laboratory, Shenzhen, China \\ ${ }^{4}$ Harbin Institute of Technology, Harbin, China \\ ${ }^{5}$ The University of Manchester, Manchester, UK
}

\begin{abstract}
The enhancement of the late sodium current $\left(I_{N a L}\right)$ has been demonstrated to contribute to the cardiac arrhythmias. However, its arrhythmogenic mechanism at the cellular and tissue level remains incompletely elucidated. In this study, the O'Hara-Rudy model of human ventricular cells was implemented for multi-level simulations. At the cellular level, the influences of the pathological enhanced $I_{N a L}$ on cardiac action potential characteristics, ion currents, intracellular concentration homeostasis and action potential duration (APD) restitution properties were simulated and analyzed. At the tissue level, a heterogeneous one dimensional (1D) strand was constructed to find out the impact of enhanced $I_{N a L}$ on APD dispersion and the vulnerable windows (VWs). The simulations revealed the role of augmenting $I_{N a L}$ in prolonging the APD, steepening the APD restitution curves, increasing the heterogeneity of the tissue and widening the VWs. Our simulation data provides a detailed mechanistic insight into the pro-arrhythmic role of the enhanced $I_{N a L}$ at both cellular and tissue levels.
\end{abstract}

\section{Introduction}

The late sodium current $\left(\mathrm{I}_{\mathrm{NaL}}\right)$ is a sustain component of the sodium current that keeps opening throughout the plateau of the cardiac action potential (AP). Although the magnitude of $\mathrm{I}_{\mathrm{NaL}}$ is normally small, it plays an important role in cardiac ion homeostasis and AP characters due to its long opening property. Moreover, many cardiac diseases such as LQT3 [1], ischemia [2] and heart failure [3] have been recognized to significantly enhance the $\mathrm{I}_{\mathrm{NaL}}$, which increases the influence of $\mathrm{I}_{\mathrm{NaL}}$ on the cardiac electrophysiology.

The enhanced $\mathrm{I}_{\mathrm{NaL}}$ is pro-arrhythmic due to several mechanisms. For the one hand, increased $\mathrm{I}_{\mathrm{NaL}}$ may result in early afterdepolarization (EAD) or delayed afterdepolarization (DAD) as a trigger for cardiac arrhythmia. Li et al. [4] claimed that $\mathrm{I}_{\mathrm{NaL}}$ is the prominent depolarizing current responsible for EAD in canine Purkinje cells. And the increase of $\mathrm{I}_{\mathrm{NaL}}$ may lead to the change of L-type calcium current $\left(\mathrm{I}_{\mathrm{CaL}}\right)$ and $\mathrm{Na}^{+}-\mathrm{Ca}^{2+}$ exchanger $\left(\mathrm{I}_{\mathrm{NCX}}\right)$, which are the major inward currents responsible for EAD in mammal ventricular cells [5]. If the enhanced $\mathrm{I}_{\mathrm{NaL}}$ further leads to the reverse model of the $\mathrm{I}_{\mathrm{NCX}}$, it may cause the overload of the intracellular calcium $\left(\left[\mathrm{Ca}^{2+}\right]_{\mathrm{i}}\right)$, triggering the DADs [6]. For the other hand, the enhanced $\mathrm{I}_{\mathrm{NaL}}$ may alternate the APD restitution property and tissue heterogeneities, then form a vulnerable substrate for cardiac re-entries. As shown in experimental data [7], the slope of APD restitution curve and ventricular tachycardia would be reduced when an inhibitor of $\mathrm{I}_{\mathrm{NaL}}$, ranolazine, was applied.

Although many mechanisms of the enhanced $\mathrm{I}_{\mathrm{NaL}}$ to cardiac arrhythmia have been proposed, it is difficult to investigate the role of merely changed $\mathrm{I}_{\mathrm{NaL}}$ without the influence of other factors from the single cell to the tissue in experiments. Therefore, we used a multiscale human ventricular model in this study, aiming to give a more detailed analysis of the mechanistic influence of the enhanced $\mathrm{I}_{\mathrm{NaL}}$ on cardiac electrophysiology and arrhythmias.

\section{Method}

The O'Hara-Rudy model of human ventricular cells [8], including all three sub-cell types: endocardial (Endo), midmyocardial (M) and epicardial (Epi) cells, was implemented for all simulations. For investigating the role of the enhanced $\mathrm{I}_{\mathrm{NaL}}$ in cardiac arrhythmia, the late sodium current was increased to 2-fold and 5-fold to compare with the normal condition (i.e. baseline). This increments of $\mathrm{I}_{\mathrm{NaL}}$ were chosen because the $\mathrm{I}_{\mathrm{NaL}}$ increased to about 2-fold in several pathological conditions such as heart failure and ischemia [9], and 5-fold is a rather large $\mathrm{I}_{\mathrm{NaL}}$ that we could analyse the variation with extreme cases. 
At the cellular level, we used the steady-state protocol to investigate the steady-state effects of the enhanced $\mathrm{I}_{\mathrm{NaL}}$ on the human ventricular myocytes. The standard simulation lasted 100s with a basic cycle length (BCL) of $1000 \mathrm{~ms}$. The S1-S2 protocol was further used for obtaining the APD restitution properties. We applied $10 \mathrm{~S} 1 \mathrm{~s}$ with a BCL of $1000 \mathrm{~ms}$ and an additional S2 with decreased diastolic intervals (DI). Then the APD evoked by the S2 was recorded to plot against the DI to get the APD restitution curves.

At the tissue level, a heterogeneous 1D strand was constructed to find out the impact of the enhanced $\mathrm{I}_{\mathrm{NaL}}$ on APD dispersion variations. The strand had a length of $150 \mathrm{~mm}$, which contained 100 nodes with a spatial resolution of $0.15 \mathrm{~mm}$. The ratio of Endo: $\mathrm{M}$ : Epi in the strand was set as 3:4:3. Again, the steady-state protocol was used in the tissue model. For each condition, the tissue model was run with a BCL of $1000 \mathrm{~ms}$ for 10 beats, then APDs for all nodes were recorded and APD spatial gradient was calculated. The vulnerable windows (VWs) were calculated by the S1-S2 protocol. We applied $10 \mathrm{~S} 1 \mathrm{~s}$ at the beginning of the strand with a BCL of 1000ms and an additional S2 at the junction of $\mathrm{M}$ and Epi cells after a time interval. The S2 would trigger three conditions: bidirectional propagated AP wave, single directional propagated AP wave and conduction block based on different time intervals. The VW then was defined as the range of the time interval that could cause the single directional propagated AP waves.

\section{Results}

\subsection{Role of the enhanced $I_{\mathrm{NaL}}$ in the human ventricular model at the cellular level}

The Endo cell was chosen for representing the cellular simulations. The steady-state effect of the enhanced $\mathrm{I}_{\mathrm{NaL}}$ on the cardiac AP characteristics, ion currents, and intracellular ion concentration homeostasis are shown in Figure 1. The most significant variation of the AP characteristics is the APD. We can see that 2-fold $\mathrm{I}_{\mathrm{NaL}}$ can increase the APD by about $12 \%$ and the 5-fold $\mathrm{I}_{\mathrm{NaL}}$ substantially lengthened the APD by $33 \%$. However, no apparent differences between the baseline and enhanced $\mathrm{I}_{\mathrm{NaL}}$ models on other AP characteristics such as resting potential or AP amplitude was observed. Meanwhile, one possible reason for the EAD and DAD triggered by the enhanced $\mathrm{I}_{\mathrm{NaL}}$ is both related to the $\mathrm{I}_{\mathrm{NCX}}$, which may reduce or even reverse during a large $\mathrm{I}_{\mathrm{NaL}}$. However, in our simulations, even 5fold $\mathrm{I}_{\mathrm{NaL}}$ only increased the $\left[\mathrm{Na}^{+}\right]_{\mathrm{i}}$ by about $0.5 \mathrm{mM}$, resulting in an insignificant reduction of the $\mathrm{I}_{\mathrm{NCX}}$. Therefore, the increase of peak $\left[\mathrm{Ca}^{2+}\right]_{\mathrm{i}}$ and $\left[\mathrm{Ca}^{2+}\right]_{\mathrm{SR}}$ is not distinct as well. This result is consistent with previous simulation study using a rabbit ventricular model, which found that the increase of $\mathrm{I}_{\mathrm{NaL}}$ did not change the $\left[\mathrm{Na}^{+}\right]_{\mathrm{i}}$.
Hence, our simulations advised that simply enhanced $\mathrm{I}_{\mathrm{NaL}}$ would not lead to the cell predispose to cardiac arrhythmia at cellular levels.
A

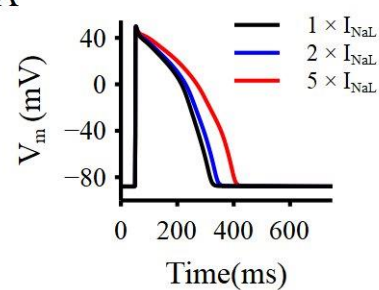

$\mathrm{C}$

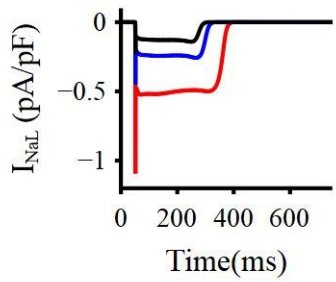

E

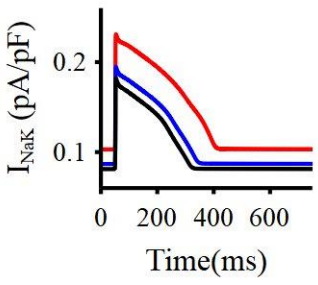

G

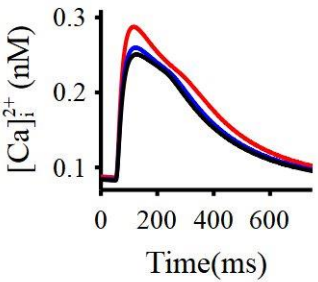

B

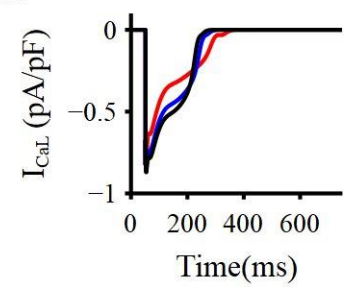

$\mathrm{D}$

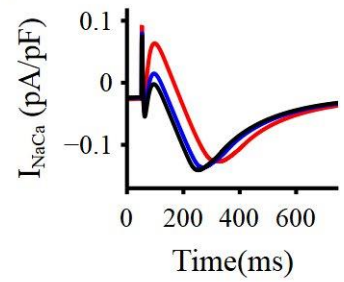

F

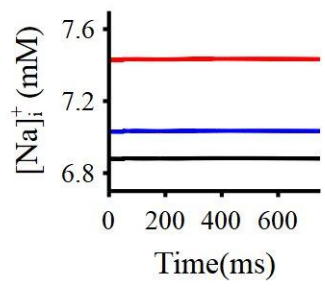

$\mathrm{H}$

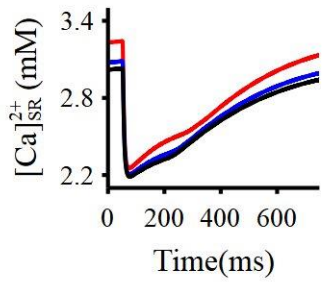

Figure 1. Action potential and ion currents in normal, 2fold $\mathrm{I}_{\mathrm{NaL}}$ and 5-fold $\mathrm{I}_{\mathrm{NaL}}$ conditions. Comparison of (A) Action potential, (B) L-type calcium channel, (C) Late sodium channel, (D) $\mathrm{Na}^{+}-\mathrm{Ca}^{2+}$ exchanger, (E) $\mathrm{Na}^{+}-\mathrm{K}^{+}$ pump, (F) intracellular $\mathrm{Na}^{+}$concentration, (G) intracellular $\mathrm{Ca}^{2+}$ transients, $(\mathrm{H}) \mathrm{Ca}^{2+}$ concentration in Sarcoplasmic Reticulum (SR) for each condition are shown in corresponding panels.

On the other hand, it worth noting that the enhanced $\mathrm{I}_{\mathrm{NaL}}$ increased the rate dependence of ventricular repolarisation as shown in Figure 2. By plotting the relationship between the APD and its preceding DI, we can get the APD restitution curve to describe the degree of APD shortens with the decrease of the AP recovery time. In our simulations, we have found that the enhancement of the $\mathrm{I}_{\mathrm{NaL}}$ would increase the slope of APD restitution curve in all three cell types, consisting with experimental findings such as in the rabbit and human myocyte [5], [10]. This 
phenomenon is also called reverse-use-dependence, which may be pro-arrhythmic with pathological decreased $\mathrm{I}_{\mathrm{Kr}}$ [11]. The reason for this reverse-use-dependence of $\mathrm{I}_{\mathrm{NaL}}$ may have two aspects: a) the $\mathrm{I}_{\mathrm{NaL}}$ would decrease with the decrease of the DI since the $\mathrm{Na}^{+}$channels can hardly recovery from the inactivation status; b) the $\mathrm{I}_{\mathrm{NaL}}$ would decrease with the decrease of the APD. In addition, steep APD restitution curve is commonly correlated with APD alternans, which is a determined pro-arrhythmic factor.
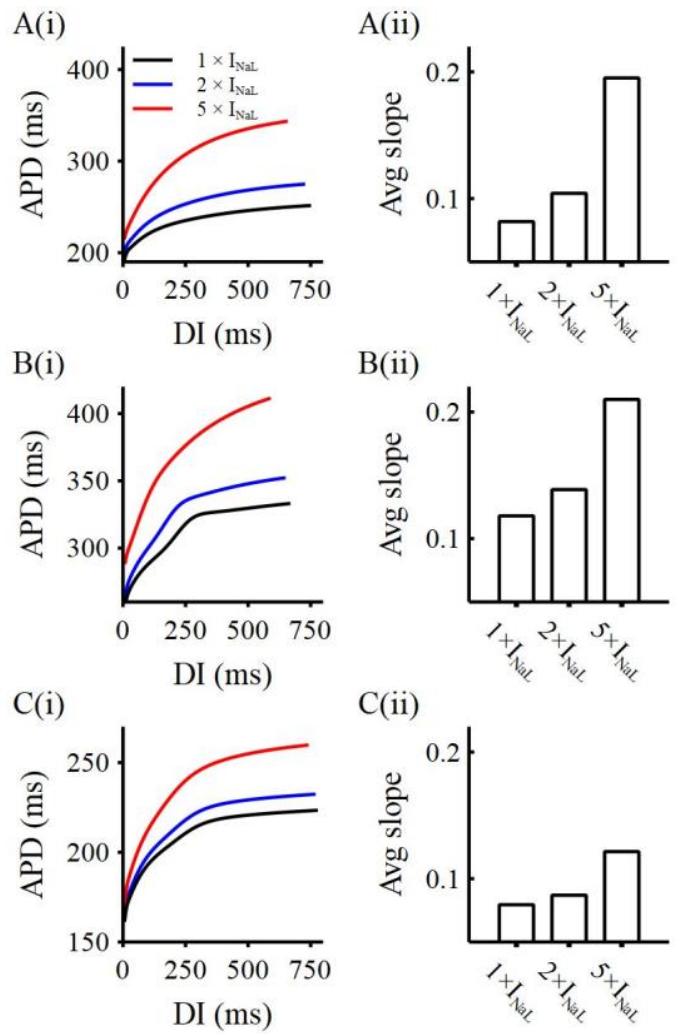

Figure 2. APD restitution properties for all three ventricular cell types in normal, 2-fold and 5-fold $\mathrm{I}_{\mathrm{NaL}}$ conditions. APD restitution curves (i) and corresponding average slopes (ii) for Endo (A), M (B) and Epi (C) cells. DI: diastolic interval.

Moreover, we noticed that the degrees of slope increment are different in three cell types. A large increment was observed in Endo and M cells, while a small increase was found in Epi cells. This demonstrated that the enhanced $\mathrm{I}_{\mathrm{NaL}}$ may lead to increased tissue heterogeneity. For verifying this idea, we have plotted the APD of all three cell types in the normal and enhanced $\mathrm{I}_{\mathrm{NaL}}$ conditions at BCL of $1000 \mathrm{~ms}$, and calculated the $\triangle \mathrm{APD}$ between them as shown in Figure 3.

Interestingly, with the increase of $\mathrm{I}_{\mathrm{NaL}}$, the APD differences between each cell types are not always increased. The $\triangle \mathrm{APD}$ between $\mathrm{M}$ and Epi cells rise with the rise of $\mathrm{I}_{\mathrm{NaL}}$, whereas the APD difference between the M and Endo cells is decreased.
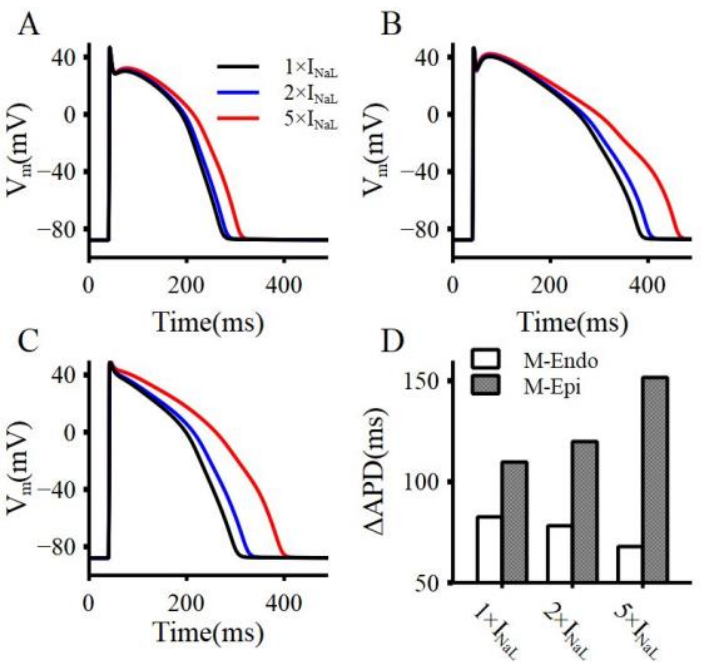

Figure 3. AP and $\triangle \mathrm{APD}$ in normal, 2-fold and 5-fold $\mathrm{I}_{\mathrm{NaL}}$ conditions. (A-C) Superimposed typical APs in three conditions for Epi (A), M (B) and Endo (C) cells. (D). $\triangle \mathrm{APD}$ between $\mathrm{M}$ and Endo cells and $\triangle \mathrm{APD}$ between $\mathrm{M}$ and Epi cells for each condition.

\subsection{Role of the enhanced $\mathrm{I}_{\mathrm{NaL}}$ in the tissue model}

For further investigating the influence of the enhanced $\mathrm{I}_{\mathrm{NaL}}$ on the APD dispersion, tissue simulations were conducted on a heterogeneous 1D stand. With a typical BCL of $1000 \mathrm{~ms}$, the APD dispersion and corresponding spatial gradient are shown in Figure 4.
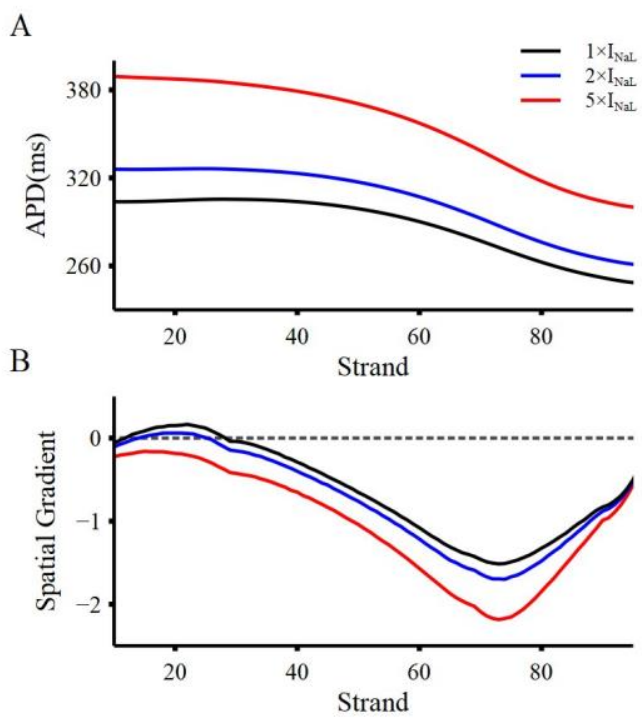

Figure 4. (A). APD dispersion of the 1D stand in normal, 2-fold and 5-fold $\mathrm{I}_{\mathrm{NaL}}$ conditions. (B). APD spatial gradient of the 1D stand in normal, 2-fold and 5-fold $\mathrm{I}_{\mathrm{NaL}}$ conditions. 
Similar to the cellular simulations, the spatial gradient around the part of Endo to $\mathrm{M}$ cells only have a minor increase (with 5-fold $\mathrm{I}_{\mathrm{NaL}}$ ) or a minor decrease (with 2-fold $\mathrm{I}_{\mathrm{NaL}}$ ) variation. However, the spatial gradient kept rising along the strand, reaching its maximum increment (increased by $13 \%$ for 2 -fold $\mathrm{I}_{\mathrm{NaL}}$ and $45 \%$ for 5 -fold $\mathrm{I}_{\mathrm{NaL}}$ ) near the junction of $\mathrm{M}$ and Epi cells.

The VW was further calculated at this maximum spatial gradient junction. The VW is a time window that a stimulus would cause unidirectional AP propagation (e.g. Figure 5B). In tissue, the unidirectional AP propagation is predisposing to reentries, which is a recognized factor that predisposes to cardiac arrhythmias. Simulation results (shown in Figure 5D) showed that the enhancement of $\mathrm{I}_{\mathrm{NaL}}$ widened the $\mathrm{VW}$, indicating that the tissue has higher possibility to generate unidirectional propagation. Furthermore, the minimum interval that caused the unidirectional propagation increased from about $335 \mathrm{~ms}$ (normal) to about $390 \mathrm{~ms}$ (5-fold $\mathrm{I}_{\mathrm{NaL}}$ ), which means reentries may happen at lower pacing rate, leading the tissue more likely to occur arrhythmias.

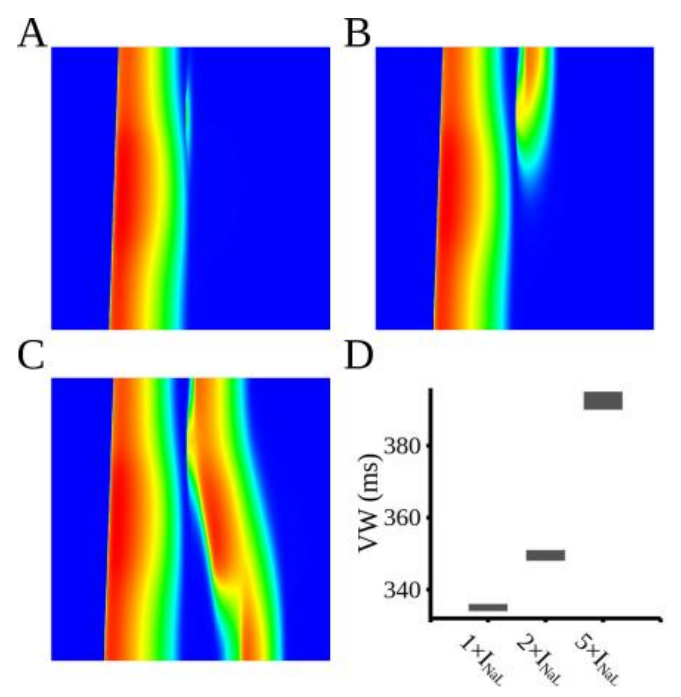

Figure 5. (A-C) AP waveform propagation types after the application of the S2 simulation: (A) a typical conduction block condition; (B) a typical unidirectional propagation condition; (C) a typical bi-directional propagation condition. (D) Calculated vulnerable window (VW) at the junction of the $\mathrm{M}$ and Epi cells in normal, 2-fold and 5-fold $\mathrm{I}_{\mathrm{NaL}}$ conditions.

\section{Conclusion}

In this study, our simulation results showed that the enhancement of $\mathrm{I}_{\mathrm{NaL}}$ mainly lengthens the APD at the cellular level, while having limited influence on the intracellular $\mathrm{Na}^{+}$concentrations. The pro-arrhythmic role of merely increased $\mathrm{I}_{\mathrm{NaL}}$ is mainly due to the increased tissue heterogeneity, especially the heterogeneity between the $\mathrm{M}$ and Epi cells, which increased the VW and led to higher possibility of the re-entry generation. These findings are consistent with previous experimental studies, adding a theoretical basis for the mechanism of cardiac arrhythmia related to the late sodium current.

\section{References}

[1] Q. Wang et al., 'SCN5A mutations associated with an inherited cardiac arrhythmia, long QT syndrome', Cell, vol. 80, no. 5, pp. 805-811, Mar. 1995.

[2] Y. Song, J. C. Shryock, and L. Belardinelli, 'A slowly inactivating sodium current contributes to spontaneous diastolic depolarization of atrial myocytes', Am. J. Physiol. Heart Circ. Physiol., vol. 297, no. 4, pp. H1254-1262, Oct. 2009.

[3] B. Horvath and D. M. Bers, 'The late sodium current in heart failure: pathophysiology and clinical relevance', ESC Heart Fail., vol. 1, no. 1, pp. 26-40, Sep. 2014.

[4] P. Li and Y. Rudy, 'A model of canine purkinje cell electrophysiology and $\mathrm{Ca}(2+)$ cycling: rate dependence, triggered activity, and comparison to ventricular myocytes', Circ. Res., vol. 109, no. 1, pp. 71-79, Jun. 2011.

[5] L. Belardinelli, W. R. Giles, S. Rajamani, H. S. Karagueuzian, and J. C. Shryock, 'Cardiac late Na+ current: proarrhythmic effects, roles in long QT syndromes, and pathological relationship to CaMKII and oxidative stress', Heart Rhythm, vol. 12, no. 2, pp. 440-448, Feb. 2015.

[6] C. Antzelevitch, V. Nesterenko, J. C. Shryock, S. Rajamani, Y. Song, and L. Belardinelli, 'The role of late $\mathrm{I}_{\mathrm{Na}}$ in development of cardiac arrhythmias', in Voltage Gated Sodium Channels, P. C. Ruben, Ed. Berlin, Heidelberg: Springer Berlin Heidelberg, 2014, pp. 137-168.

[7] N. Morita et al., 'Suppression of re-entrant and multifocal ventricular fibrillation by the late sodium current blocker ranolazine', J. Am. Coll. Cardiol., vol. 57, no. 3, pp. 366375, Jan. 2011.

[8] T. O'Hara, L. Virág, A. Varró, and Y. Rudy, 'Simulation of the undiseased human cardiac ventricular action potential: model formulation and experimental validation', PLoS Comput. Biol., vol. 7, no. 5, p. e1002061, May 2011.

[9] K. Cardona, B. Trenor, and W. R. Giles, 'Changes in intracellular $\mathrm{Na}^{+}$following enhancement of late $\mathrm{Na}^{+}$current in virtual human ventricular myocytes', PLOS ONE, vol. 11, no. 11, p. e0167060, Nov. 2016.

[10] D. Guo et al., 'Contribution of late sodium current ( $\left.\mathrm{I}_{\mathrm{Na}-\mathrm{L}}\right)$ to rate adaptation of ventricular repolarization and reverse usedependence of QT-prolonging agents', Heart Rhythm, vol. 8, no. 5, pp. 762-769, May 2011.

[11] L. Wu et al., 'Late sodium current contributes to the reverse rate-dependent effect of $\mathrm{I}_{\mathrm{Kr}}$ inhibition on ventricular repolarization', Circulation, vol. 123, no. 16, pp. 1713 1720, Apr. 2011.

Address for correspondence:

Wei Wang

HIT Campus of University Town of Shenzhen,

Shenzhen, 518055, China.

wangwei2019@hit.edu.cn 